\title{
PROPOSTAS INOVADORAS NA FORMAÇÃO DO PROFISSIONAL PARA O SISTEMA ÚNICO DE SAÚDE
}

\author{
INNOVATIVE PROPOSALS IN VOCATIONAL TRAINING FOR THE UNIFIED HEALTH SYSTEM
}

\author{
Dinair Leal da Hora ${ }^{1}$ \\ Regina Maria de Carvalho Erthal ${ }^{2}$ \\ Claudia Teresa Vieira de Souza ${ }^{3}$ \\ Eloísa Leal da Hora ${ }^{4}$
}

Resumo Este ensaio apresenta uma discussão a respeito dos novos cenários da formação do profissional da saúde para o Sistema Único de Saúde (SUS) e sua relação com as propostas apresentadas no relatório Health Professionals for a New Century: Transforming Education to Strengthen Health Systems in an Interdependent World. O relatório foi elaborado por uma comissão independente formada por 18 profissionais e acadêmicos de vários países, liderada pelos médicos e professores Julio Frenk e Lincoln Chen, criada em janeiro de 2010, nos Estados Unidos pelo setor de saúde para homenagear o centenário do Relatório Flexner. A finalidade do relatório foi apresentar recomendações para a adoção de inovações educacionais e institucionais voltadas para a formação de uma nova geração de profissionais mais bem equipados para lidar com os desafios presentes e futuros da área, numa perspectiva global de promoção da saúde. Essa discussão resultou na percepção de que, apesar de políticas e programas governamentais criados no Brasil desde 2001, e de iniciativas institucionais pontuais, a formação dos profissionais da área da saúde ainda é fortemente orientada por uma concepção pedagógica hospitalocêntrica que categoriza os adoecimentos por critérios biologicistas e que dissocia clínica e política, o que não é adequado para contribuir para o fortalecimento do SUS.

Palavras-chave formação profissional em saúde; organização curricular em saúde; educação e saúde.
Abstract This essay discusses the new scenarios for the training of health professionals for the Sistema Único de Saúde (SUS, Unified Health System) and how they are related the proposals made in the report entitled "Health Professionals for a New Century: Transforming Education to Strengthen Health Systems in an Interdependent World". It was drafted by an independent committee made up of 18 professionals and academics from several countries, led by physicians and professors Julio Frenk and Lincoln Chen and created in January 2010, in the United States, by the health sector to honor the centennial of the Flexner Report. The purpose of the report was to lay down recommendations for the adoption of educational and institutional innovations aimed at training a new generation of professionals in order for them to be best equipped to deal with the area's present and future challenges in a comprehensive perspective of health promotion. The discussion resulted in the perception that, despite governmental policies and programs created in Brazil since 2001 and of timely institutional initiatives, health area professionals still get training that is strongly guided by a hospital-centric educational conception, categorizes illnesses based on biological criteria, and dissociates clinic practice and politics, something that is not suitable to help strengthen the SUS.

Keywords training of health professionals; curriculum organization in health; education and health. 


\section{Introdução}

As importantes reformas nos processos de formação dos profissionais da saúde ocorrem primordialmente nas propostas para a educação médica que, depois, são transferidas para o ensino da odontologia, da enfermagem, da farmácia e das demais carreiras da área da saúde, o que se configura na constituição de uma concepção médico-hegemônica no conjunto dos cursos da área da saúde e na definição do perfil desses profissionais no exercício de suas práticas (Ceccim e Capozzolo, 2004).

O modelo de educação médica proposto por Abraham Flexner em 1910, na publicação do estudo intitulado Medical Education in the United States and Canada: a report to the Carnegie Foundation for the advancement of theaching (Flexner, 1910), é reconhecido como a mais importante reforma do processo de formação dos profissionais da medicina nos Estados Unidos. A relevância das recomendações de Flexner foi de tal impacto que trouxe profundas transformações na formação médica e na medicina em âmbito mundial, inclusive sendo replicadas nas outras profissões da área da saúde.

A proposta de Flexner, embora tenha recebido ao longo do tempo fortes críticas, especialmente porque nunca conseguiu atender às necessidades de saúde das sociedades nas quais foi implantada (Tomey, 2002), ainda se encontra em vigor na maior parte das escolas médicas do mundo, onde permanece organizando seus currículos: um ciclo inicial de disciplinas básicas realizadas nos laboratórios e outro ciclo de estudos clínicos em hospitais e centros médicos acadêmicos. Os hospitais são, assim, o locus privilegiado de transmissão do conhecimento médico, restando às faculdades o ensino de laboratório nas áreas básicas e a parte teórica das especialidades (Pagliosa e Da Ros, 2008), em uma reforma marcada pela introdução da profissionalização do ensino em saúde que admitiu a hierarquização das aprendizagens - do básico ao clínico - e a aprendizagem em ambientes de concentração de quadros nosológicos - modelo hospitalocêntrico. Essa mudança foi trazida por Flexner (Tomey, 2002). Dois dos críticos do modelo flexneriano destacam:

\footnotetext{
O paradigma flexneriano é coerente com o conceito de saúde como ausência de doença e constitui uma âncora que permite sustentar a prática sanitária da atenção médica. Para superá-lo há que se buscar a construção de um novo paradigma que permita dar conta da saúde vista em sua positividade e como um processo que pode melhorar ou deteriorar conforme a ação da sociedade sobre os fatores que lhe são determinantes, sobre o estado de saúde acumulado e sobre as consequências da perda da saúde (Ferreira e Buss, 2002, p. 31).
}

A formação dos profissionais de saúde ainda se encontra fechada aos avanços da cidadania, que exige da sociedade um novo posicionamento: 
saúde como direito de todos e dever do Estado; saúde como direito à vida com qualidade; saúde como direito à atenção integral, com privilégio da promoção e prevenção, sem prejuízo da recuperação e da reabilitação dos estados de saúde; e saúde como expressão do desenvolvimento da vida. As dificuldades para organizar propostas de formação mais flexíveis e qualitativas são provocadas por vários fatores estruturais e conjunturais, entre os quais é possível citar a interferência das ações dos grupos corporativos e produtivos das redes de saúde; as lutas de poder nos espaços laborais; a inconsistência da qualidade dos processos formativos dos professores, pela insuficiência da infraestrutura dos espaços de aprendizagem e de articulação entre o ensino, o serviço e a gestão das atividades assistenciais à saúde (Ceccim e Carvalho, 2004).

Este texto propõe uma discussão a respeito dos novos cenários da formação do profissional da saúde para o Sistema Único de Saúde (SUS) e sua relação com as propostas apresentadas pelo relatório Health Professionals for a New Century: Transforming Education to Strengthen Health Systems in an Interdependent World (Os profissionais de saúde para um novo século: educação transformadora para fortalecer os sistemas de saúde em um mundo interdependente), elaborado em 2010 por profissionais e acadêmicos de vários países.

\section{Os atuais desafios para a formação do profissional da saúde}

Em novembro de 2010, veio a público o relatório intitulado Health Professionals for a New Century: Transforming Education to Strengthen Health Systems in an Interdependent World, resultado do trabalho realizado pela Comissão Independente sobre a Educação dos Profissionais de Saúde para o Século XXI, liderada pelos médicos e professores Julio Frenk e Lincoln Chen com a participação de mais 18 profissionais e acadêmicos de vários países.

Essa Comissão, criada pelos setores de saúde dos Estados Unidos em janeiro de 2010 para homenagear o centenário do Relatório Flexner, teve como tarefa recomendar, com base na adoção de uma perspectiva global de promoção da saúde, "inovações educacionais e institucionais voltadas para a formação de uma nova geração de profissionais melhor equipados para lidar com os desafios presentes e futuros na área" (RETS, 2011, p. 3).

Trabalhando com a realidade de um espaço de saúde globalizado, com aumento significativo da expectativa de vida no século $\mathrm{XX}$, atribuído ao avanço dos conhecimentos e melhoria das condições de vida, a comissão chama a atenção para o fato de que o conhecimento científico, a par de produzir novas tecnologias, habilita os indivíduos na adoção de estilos de vida saudáveis, na busca de cuidados, na percepção de seus direitos e na atenção aos determinantes e condicionantes da saúde. 
O estudo realizado pela Comissão Independente centrou-se nos profissionais da saúde, concluintes de cursos superiores em universidades e instituições legalmente autorizadas, nas carreiras de medicina, enfermagem, obstetrícia e de saúde pública. Entretanto, tem como alvo todos os trabalhadores da saúde, sujeitos a processos educativos que visam desenvolver conhecimentos, habilidades e valores que possam melhorar a saúde das populações (Frenk \& Chen, 2010).

Conforme aponta o relatório, a Comissão Independente, para alcançar seus objetivos, procurou trabalhar dentro de uma visão global, de uma perspectiva multiprofissional e de uma abordagem sistêmica, utilizando dados obtidos com base em uma revisão de trabalhos publicados, complementada por consultas aos especialistas da área e a jovens profissionais, adotando os seguintes procedimentos:

- análise de todos os artigos relevantes para a educação em medicina, enfermagem e saúde pública, indexados no PubMed e no Medline;

- compilação dos dados sobre instituições de ensino de graduação em medicina nas bases da Foundation for the Advancement of International Medical Education and Research (Faimer) e da Avicenna, atualizados com dados regionais e nacionais mais recentes;

- análise de dados de websites sobre saúde pública;

- estimativas de financiamento, calculadas por meio de micro e macroabordagens: no caso da educação médica e da enfermagem, o custo total foi calculado multiplicando-se o custo unitário de graduação pelo número de diplomados. Os resultados obtidos foram comparados com o percentual dos recursos destinados a essas áreas.

Os autores do estudo chamam a atenção para os seguintes fatos: no caso da obstetrícia, não foram encontrados dados internacionais; o número de graduados nas carreiras de medicina, obstetrícia e enfermagem foi obtido em relatórios e outros documentos; não foi possível estimar o número de diplomados em saúde pública devido à ausência de dados e de uma definição única do curso. Os autores argumentam ainda que, apesar da relativa imprecisão, os dados obtidos foram capazes de fornecer alguma garantia da ordem de grandeza das estimativas.

Entre as principais conclusões, o estudo constatou que, no ano de sua realização, 2010, havia em todo o mundo 2.420 escolas de medicina, 476 escolas ou departamentos de saúde pública e um número indeterminado de instituições educacionais de nível superior de enfermagem, formando cerca de um milhão de novos médicos, enfermeiros, parteiras e profissionais de saúde pública a cada ano.

Além do número de instituições formadoras de profissionais da saúde para atender às demandas globais, foi possível constatar sua má distribuição tanto entre países como dentro deles: quatro países (China, Índia, Brasil e Estados Unidos) têm, cada um, mais de 150 escolas médicas, enquanto 36 
não têm nenhuma escola médica. Vinte e seis países da África subsaariana têm uma ou nenhuma escola médica. Diante de tais desequilíbrios, não é surpreendente que as faculdades de medicina não consigam, nesse contexto, desempenhar satisfatoriamente suas atribuições de realizar um atendimento de qualidade em países onde se encontram populações assoladas pelo pesado fardo das doenças infecciosas emergentes e reemergentes.

Quando analisaram o aspecto financeiro, os autores da pesquisa constataram que a despesa global destinada à formação de profissionais de saúde é de cerca de US\$ 100 bilhões por ano, novamente com grandes disparidades entre os países. Este montante é menos de $2 \%$ dos gastos com saúde em todo o mundo e extremamente modesto para um trabalho intensivo.

Nos achados dos autores, a fragilidade e a irregularidade dos processos de acreditação e de certificação institucionais e dos sistemas de aprendizagem estão presentes em todo o mundo. E sua análise demonstrou a escassez de informações e pesquisas sobre saúde e educação profissional.

Embora muitas instituições educacionais em todas as regiões tenham tomado iniciativas inovadoras para fortalecer e transformar os processos de formação dos profissionais da saúde, os componentes da comissão, em seu estudo, constataram que são muito reduzidas as evidências robustas disponíveis sobre a efetividade dessas reformas.

Em relação aos movimentos das reformas no ensino médico, Frenk e Chen destacam que

Três gerações de reformas educacionais caracterizam o progresso durante o século passado. A primeira geração, lançada no início do século XX, ensinou um currículo baseado na ciência. Cerca de meados do século, a segunda geração introduziu inovações baseadas em práticas instrucionais. Para avançar na terceira geração de reformas, a Comissão apresenta a visão de que os profissionais de saúde em todos os países devem ser educados para mobilizar conhecimentos e participar de raciocínio crítico e conduta ética, de modo que sejam competentes para se relacionar com o paciente e com os sistemas de saúde e com a população como membros de equipes de resposta local e globalmente conectadas. O objetivo final é assegurar a cobertura universal dos serviços abrangentes de alta qualidade, que são essenciais para se avançar para a equidade em saúde dentro e entre países (Frenk e Chen, 2010, p. 1.924, tradução nossa). ${ }^{5}$

Ao propor uma reforma educacional, a comissão aponta para a necessidade de se trabalhar em uma perspectiva multiprofissional e com uma abordagem sistêmica, considerando fundamental a interdependência dos sistemas de educação e saúde, em que a população serve de base aos dois sistemas. Nesse modelo, parte-se da necessidade das populações/indivíduos, que se transformam em demandas para os serviços de saúde e educação que 
são responsáveis por suprir tal demanda com profissionais qualificados para atuar nos serviços de saúde de forma multidisciplinar. Nessa cadeia, a comissão considera que a população não pode ser vista como mera usuária dos sistemas, mas como coprodutora deles (RETS, 2011), e que o espaço educativo é formador de agentes de mudanças.

Assim, a Comissão julga fundamental a articulação entre os sistemas de educação e de saúde e a percepção das pessoas no centro dos processos de produção e direção das necessidades e demandas de ambos os sistemas. Considera que os serviços educacionais têm a responsabilidade de prover os serviços de saúde com uma força de trabalho bem formada pelo desenvolvimento de novas estratégias pedagógicas e institucionais. Para tal, apresenta propostas de uma reforma de ensino que tenha ampla abrangência, desde a admissão até a conclusão do curso, de modo a promover a melhoria dos sistemas de atenção para que atendam às necessidades da população de forma equitativa e eficiente. São elas:

- adoção de currículos baseados em competências, capazes de mudar rapidamente para atender às demandas dos sistemas de saúde, substituindo os planos de cursos estáticos;

- promoção da educação interprofissional e transprofissional, como forma de reduzir o corporativismo, ao mesmo tempo reforçando as relações colaborativas não hierárquicas em equipes eficazes;

- exploração do potencial da tecnologia da informação para a aprendizagem; - adaptação de recursos globais para enfrentar os desafios locais, compartilhando experiências, currículos, corpo docente, materiais didáticos e intercâmbio estudantil;

- fortalecimento dos recursos educacionais, incluindo professores, conteúdos programáticos, material didático e infraestrutura;

- promoção de um novo profissionalismo, cujos critérios objetivos para a classificação dos profissionais da saúde sejam as competências, transformando as atuais corporações.

Em relação às reformas das instituições responsáveis pela formação dos profissionais da saúde e pelo fomento dessa formação, a comissão considera essencial a adoção de um esforço nacional de planejamento conjunto entre os setores de saúde e de educação, envolvendo todos os interessados na busca do fortalecimento mútuo, da promoção da cultura da investigação crítica e da argumentação pública. Para tal, propõe:

- criação de procedimentos de planejamento coletivo em cada país, reunindo ministérios da Saúde e da Educação, associações profissionais e comunidade acadêmica, a fim de superar a fragmentação via avaliação das condições nacionais, definição de prioridades, formulação de políticas, controle das mudanças e harmonização entre oferta e demanda de profissionais de saúde para atender às necessidades da população; 
- expansão de centros acadêmicos para sistemas acadêmicos que atuem de forma mais colaborativa e responsável na questão da educação continuada; - criação de redes, alianças e consórcios interinstitucionais de ensino em todo o mundo e com governos, organizações da sociedade civil, empresas e meios de comunicação;

- naturalização de uma cultura de investigação crítica nas universidades e em outras instituições de formação, a fim de mobilizar os conhecimentos científicos, a deliberação ética e o debate público, gerando assim uma transformação social esclarecida.

Os componentes da comissão reconhecem as dificuldades para a implantação de suas propostas e afirmam a necessidade de superarem as barreiras que lhes serão impostas pela mobilização das lideranças, pelo reforço dos investimentos, pela definição de políticas e de incentivos, pelo alinhamento da acreditação e pelo reforço da aprendizagem global.

As análises trazidas pela Comissão apontam para a construção de um projeto educativo contra-hegemônico com a inserção de conteúdos/temas de políticas de saúde nos currículos da formação em saúde, numa abordagem que articule essas políticas a estratégias promovidas pela dinâmica dos movimentos sociais contemporâneos, evidenciando tensões e conflitos nesse processo. Ou seja: conhecimentos que discutam a configuração das tendências do Estado contemporâneo e as formas de adesão ao movimento do capital na atual formação histórica (Pereira, 2008).

Assim, é possível pensar na aplicação da racionalidade e dos conhecimentos sobre as doenças numa perspectiva emancipatória, reconhecendo que as propostas de intervenção dos profissionais da saúde podem alargar os modos de cuidar da vida, o que não decorre, necessariamente, da mera aplicação do melhor conhecimento científico disponível (Mattos, 2008).

Dessa forma, é preciso operar uma proposta curricular contra-hegemônica, rompendo a organização e a execução de grades curriculares que agudizam a dicotomia entre a teoria e a prática, isto é, ensinando primeiro os conteúdos teóricos e depois indo para a realidade concreta de forma desarticulada, a favor de

Uma educação emancipatória para a autonomia [que] requer uma formação cada vez mais significativa e consciente, durante toda a vida dos indivíduos. Reafirma-se aqui a importância da consistência do trabalho educativo numa perspectiva de democracia, conjunto e compromisso (Freire, 2011, p. 58),

construída de conteúdos e práticas que evidenciem o trabalho em saúde como prática social privilegiada. Assim, com base no entendimento de que os seres humanos são as relações que estabelecem entre si, a solidariedade com o outro, a autonomia para tomada de decisões participativas, a 
preocupação e o sentido de cuidar do outro constroem o projeto civilizatório de humanidade.

\section{Os cenários para a formação profissional em saúde no Brasil}

No Brasil, as lutas sociais em favor de uma reforma sanitária que garantisse a saúde como um direito de cidadania foram consagradas na Constituição de 1988, cuja institucionalização deu-se pelo SUS, que representa o maior mercado empregador do profissional da saúde e a maior concentração e diversificação de processos de aprendizagem da área (Pinheiro, Ceccim e Mattos, 2005).

A Constituição estabeleceu que as ações e os serviços de saúde, ao se constituírem por um sistema único, integram uma rede que deve ser organizada segundo três diretrizes: universalidade, integralidade e equidade. Definiu também que cabe ao SUS ordenar a formação de recursos humanos na área de saúde. Assim, o perfil de formação desses profissionais assenta-se no desenvolvimento de sua capacidade de oferecer atenção integral - fundamental para a conquista da integralidade na atenção à saúde. As diretrizes constitucionais do SUS devem ser tomadas como determinantes na formulação da política desse sistema para a mudança na sua formação básica, com a assunção de estratégias didático-pedagógicas ou modos de ensinar problematizadores, construtivistas ou com protagonismo ativo dos estudantes (Ceccim e Feuerwerker, 2004a).

Várias ações foram adotadas para buscar a concretização de inovações educacionais nos cursos de graduação de modo a que os princípios do SUS fossem alcançados. Entre essas ações, pode-se destacar a transformação na orientação e na organização dos cursos, via construção de novas diretrizes curriculares nacionais para as profissões da área de saúde, que preconizavam a ruptura do modelo de currículo mínimo obrigatório para as carreiras, como possibilidade para a perspectiva transformadora da formação de profissionais de saúde.

No contexto das reformas educacionais realizadas no Brasil a partir de meados da década de 1990, novas diretrizes curriculares foram elaboradas para os cursos de graduação no Brasil, o que levou alunos, professores e funcionários das instituições de ensino superior a discutirem, em maior ou menor medida, as mudanças contidas nessas diretrizes.

Para os cursos da área de saúde, as diretrizes curriculares homologadas em sua maioria nos anos de 2001 e 2002 são resultantes da disputa entre diferentes ideologias, especialmente entre as daqueles que defendiam o desenvolvimento das disciplinas em ambientes hospitalares e intramuros das universidades e as concepções daqueles que, assim como indicavam as no- 
vas diretrizes, defendiam uma formação voltada para o campo da atenção primária à saúde (Rossoni e Lampert, 2004).

Aprovadas, em sua maioria, entre 2001 e 2002, as diretrizes curriculares nacionais dos cursos de graduação em saúde (exceto Medicina Veterinária, Psicologia, Educação Física e Serviço Social) afirmaram que a formação do profissional de saúde deve contemplar o sistema de saúde vigente no país, o trabalho em equipe e a atenção integral à saúde (formação de biomédicos, fisioterapeutas, fonoaudiólogos, médicos, odontólogos e terapeutas ocupacionais). Reafirmando a posição de orientação ao sistema de saúde vigente, algumas profissões destacaram o SUS. É o caso da formação de farmacêuticos, em que constou o aposto com ênfase no SUS, e da formação de nutricionistas e de enfermeiros, nas quais constou ainda que a formação do profissional deve atender às necessidades sociais da saúde, com ênfase no SUS. Na profissão de enfermeiros constou o acréscimo de que o atendimento às necessidades sociais de saúde deve ser assegurado pela integralidade da atenção e pela qualidade e humanização do atendimento (Almeida, 2003, grifos do autor, p. 83).

Assim, com as mudanças propostas para os currículos dos cursos das áreas da saúde, impôs-se para as instituições de ensino superior o desafio de realizar uma formação generalista, humanista, crítica e reflexiva, em que os profissionais sejam capazes de atuar na integralidade da atenção à saúde e em equipe multiprofissional, características exigidas para atender aos princípios do SUS - como é possível perceber, por exemplo, nas diretrizes curriculares nacionais dos cursos de medicina.

Art. $3^{\circ}$ - O Curso de Graduação em Medicina tem como perfil do formando egresso/profissional, o médico, com formação generalista, humanística, crítica e reflexiva, capacitado a atuar, pautado em princípios éticos, no processo de saúde-doença em seus diferentes níveis de atenção, com ações de promoção, prevenção, recuperação e reabilitação à saúde, na perspectiva da integralidade da assistência, com senso de responsabilidade social e compromisso com a cidadania, como promotor da saúde integral do ser humano (Brasil, 2001).

Para as universidades, parece que as diretrizes curriculares constituíram-se apenas em recomendações, já que essas instituições formadoras têm autonomia didática. Por isso, em 2003, o Conselho Nacional de Saúde aprovou como política pública a iniciativa denominada Política de formação e desenvolvimento para o SUS: caminhos para a educação permanente em saúde (Brasil, 2003). Este documento foi o marco para a definição do campo de saberes e práticas da educação e ensino da saúde e para o reconhecimento de sua origem no campo da saúde coletiva, especialmente por articular 
ações interdisciplinares para a formação profissional e para a assistência em saúde.

Essa política de formação buscava reforçar a aproximação efetiva entre os sistemas de saúde e de ensino e, para tal, constituiu programas como o AprenderSUS, 6 cuja finalidade é a de aprofundar as relações de cooperação entre o sistema de saúde e as instituições de educação superior, e o Ver SUS, 7 que consiste em proporcionar aos estudantes vivência e estágio na realidade do SUS, de modo a que possam conhecer os desafios e as conquistas do sistema, aprofundar a discussão sobre o trabalho em equipe, a gestão, a atenção, a educação e o controle social organizado de diferentes formas nas várias regiões do país (Ceccim e Bilibio, 2002).

Muitas iniciativas do setor possibilitaram o desenvolvimento de um pensamento crítico e estimularam o fortalecimento do movimento por mudanças no processo de formação. Programas como os de Interiorização do Trabalho em Saúde (Pits), de Incentivo às Mudanças Curriculares nos Cursos de Medicina (Promed), de Capacitação e Formação em Saúde da Família, de Profissionalização dos Trabalhadores da Área de Enfermagem (Profae), de Aperfeiçoamento ou Especialização de Equipes Gestoras, de Formação de Conselheiros de Saúde, entre outros, caminharam nessa direção e possibilitaram a mobilização de pessoas e instituições no sentido de uma aproximação entre instituições formadoras e ações e serviços do SUS (Ceccim e Feuerwerker, 2004b).

A Política Nacional de Promoção da Saúde ratifica o compromisso do Ministério da Saúde na ampliação e qualificação das ações de promoção da saúde nos serviços do SUS. Nesse sentido, em 3 de novembro de 2005, foi assinado documento denominado Pró-Saúde (Programa Nacional de Reorientação da Formação Profissional em Saúde), que representa importante pacto intersetorial já consolidado entre os ministérios da Saúde e da Educação. Esse programa prevê a educação permanente dos profissionais da área da saúde, incorporando aos seus programas a substituição do modelo tradicional do cuidado em saúde, historicamente centrado no atendimento hospitalar e na doença, para um modelo mais humanizado com foco maior nas ações de prevenção.

O Pró-Saúde configura-se como uma estratégia de governo com ênfase na formação de profissionais de saúde, assumindo como objetivo geral o incentivo às transformações no processo de formação, de geração de conhecimentos e de prestação de serviços à população, para uma abordagem integral do processo saúde-doença.

O que se busca é a intervenção no processo formativo para que os programas de graduação possam deslocar o eixo da formação - centrado na assistência indi- 
vidual prestada em unidades especializadas - por um outro processo em que a formação esteja sintonizada com as necessidades sociais, calcada na proposta de hierarquização das ações de saúde. Além disso, que essa formação leve em conta as dimensões sociais, econômicas e culturais da população, instrumentalizando os profissionais para a abordagem dos determinantes de ambos os componentes do binômio saúde-doença da população na comunidade e em todos os níveis do sistema.

A educação dos profissionais de saúde deve ser entendida como processo permanente, que se inicia durante a graduação e é mantido na vida profissional, mediante o estabelecimento de relações de parceria entre as instituições de educação superior, os serviços de saúde, a comunidade, as entidades e outros setores da sociedade civil.

A formação do profissional em saúde, respeitando as diretrizes nacionais aprovadas pelo MEC, deve estar atenta ao acelerado ritmo de evolução do conhecimento, à mudança do processo de trabalho em saúde, às transformações nos aspectos demográficos e epidemiológicos, tendo como perspectiva o equilíbrio entre excelência técnica e relevância social (Brasil, 2005, p. 47).

Assim, a criação do Pró-Saúde veio para promover a integração ensino-serviço, visando à reorientação da formação profissional, assegurando uma abordagem integral do processo saúde-doença com ênfase na atenção básica, promovendo transformações nos processos de geração de conhecimentos, ensino e aprendizagem e de prestação de serviços à população, caracterizando-se como um movimento importante para a reorganização das instituições de ensino superior na área da saúde.

\section{Considerações em processo}

É possível perceber que as iniciativas interministeriais - Ministério da Saúde e Ministério da Educação - articuladas ao Conselho Nacional de Saúde tomadas no Brasil para a consolidação de uma política nacional de educação em saúde, de modo a consolidar o SUS na sua competência de ordenar a formação de recursos humanos na área da saúde (Brasil, 1988), aproximam-se, em grande medida, das indicações apresentadas pela Comissão Independente de 2010.

A respeito das sugestões relativas às reformas institucionais sugeridas pela comissão, destacam-se as ações conjuntas em torno da gestão do trabalho e da educação na saúde para valorizar o processo ensino-serviço, a humanização da atenção e a ampliação da concepção e da prática da integralidade e para desenvolver um sistema de educação permanente dos trabalhadores de saúde, conforme as necessidades da população e de fortalecimento do SUS. 
Uma das mais enfáticas e importantes sugestões apontadas pelos coordenadores da Comissão Independente de 2010, Julio Frenk e Lincoln Chen, refere-se aos processos de organização curricular para os cursos de formação dos profissionais da saúde com base em concepções emancipatórias, privilegiando a prática social, de modo a articular organicamente a integração ensino-serviço.

Relacionada a essa relevante sugestão apresentada pela Comissão Independente está a implantação do Pró-Saúde, cuja finalidade é a reorganização da formação profissional, assegurando uma abordagem integral do processo saúde-doença com ênfase na atenção básica, promovendo transformações nos processos de geração de conhecimentos, ensino, aprendizagem e prestação de serviços à população. Seus fundamentos estão em três dimensões: - orientação teórica, com base nos determinantes de saúde e doença, na pesquisa ajustada à realidade local e na educação permanente;

- cenários de prática, privilegiando a integração ensino-serviço, a utilização dos diversos níveis de atenção e a integração dos serviços das instituições de ensino superior (IES) com os serviços de saúde;

- orientação pedagógica, assentada na integração básico-clínica, na análise crítica dos serviços e na aprendizagem ativa. 8

Entretanto, a formação dos profissionais da área da saúde ainda é fortemente orientada por uma concepção pedagógica que estabelece o centro das aprendizagens no hospital universitário, hierarquiza os adoecimentos em critérios biologicistas e dissocia clínica e política (Ceccim e Feuerwerker, 2004a), conforme se pode verificar nas propostas curriculares, por exemplo, do curso de medicina da Universidade de São Paulo (USP), em que as disciplinas básicas estão concentradas nos primeiros semestres e as práticas hospitalares nos semestres finais, inclusive com carga horária maior.

No curso de enfermagem da Universidade Federal de Minas Gerais (UFMG), nota-se que dos nove períodos previstos para a integralização do curso, nos cinco primeiros são realizadas atividades práticas de 75 horas, e a partir do sexto semestre são realizadas atividades de estágio, num total de 565 horas-aula. 9

Dessa forma, o ensino superior na saúde ainda não se apresenta adequado para contribuir para o fortalecimento do SUS. A esse respeito o sistema enfrenta diversos desafios referentes à distribuição e fixação de profissionais, qualificação profissional, alteração do modelo de atenção exemplificado pela Estratégia Saúde da Família, alteração dos dados demográficos e epidemiológicos e modificação das necessidades e demandas populacionais por atenção à saúde. A conjunção desses aspectos, associando-se às mudanças no mundo do trabalho e na sociedade contemporânea, indica a urgente necessidade de articulação entre a formação profissional e a organização do SUS (Haddad et al., 2010). 
A formação dos profissionais de saúde como um projeto educativo extrapola a educação para o domínio técnico-científico da profissão, estendendo-se pelos aspectos estruturantes de relações e de práticas em todos os componentes de interesse ou relevância social que contribuam para a elevação da qualidade de saúde da população, tanto no enfrentamento dos aspectos epidemiológicos do processo saúde-doença quanto nos aspectos de organização da gestão setorial e estruturação do cuidado à saúde (Ceccim e Feuerwerker, 2004b).

Um dos aspectos que precisam ser discutidos e analisados é o interesse em se alcançar a compreensão interpretativa do discurso e das práticas educativas desenvolvidas nos programas estudados de formação de profissionais da saúde, dos seus documentos constitutivos e dos seus significados para os sujeitos sociais participantes do processo: gestores, estudantes, professores, que são os principais atores dos processos de formação dos profissionais de saúde.

Por meio de uma abordagem crítica no processo de aprendizagem, será possível revelar as consentaneidades e as contradições existentes entre concepções, impressões e concreticidades presentes nas propostas e nas práticas dos cursos de formação na área da saúde, assinalando suas causas, consequências e relações, de modo a se realizar o aprofundamento da compreensão dessa realidade e de suas intencionalidades e assim compreender os atos, fatos e fenômenos nela construídos. Porém, não é possível ignorar as inovações que vêm sendo realizadas por muitas instituições formadoras que, embora pontuais, têm obtido importantes avanços no rumo de uma prática mais consistente e efetiva.

\section{Notas}

1 Instituto de Pesquisa Clínica Evandro Chagas, Fundação Oswaldo Cruz , Rio de Janeiro, RJ, Brasil.

Doutora em Educação pela Universidade Estadual de Campinas.

$<$ dinair.hora@ipec.fiocruz.br>

Correspondência: Rua João Alfredo, 37, apto. 504, CEP 20511-390, Tijuca, Rio de Janeiro, RJ, Brasil.

2 Instituto de Pesquisa Clínica Evandro Chagas, Fundação Oswaldo Cruz, Rio de Janeiro, RJ, Brasil.

Doutora em Saúde Pública pela Escola Nacional de Saúde Pública Sergio Arouca, da Fundação Oswaldo Cruz. <regina.erthal@ipec.fiocruz.br> 
3 Instituto de Pesquisa Clínica Evandro Chagas, Fundação Oswaldo Cruz, Rio de Janeiro, RJ, Brasil.

Doutora em Saúde Pública pela Escola Nacional de Saúde Pública Sergio Arouca, Fundação Oswaldo Cruz. <claudia.souza@ipec.fiocruz.br>

4 Instituto de Pesquisa Clínica Evandro Chagas, da Fundação Oswaldo Cruz, Rio de Janeiro, RJ, Brasil.

Graduada em Museologia pela Escola de Museologia da Universidade Federal do Estado do Rio de Janeiro. <eloisa.hora@ipec.fiocruz.br>

${ }^{5}$ No original: "Three generations of educational reforms characterize progress during the past century. The first generation, launched at the beginning of the 20th century, taught a science-based curriculum. Around the mid-century, the second generation introduced problem-based instructional innovations. A third generation is now needed that should be systems based to improve the performance of health systems by adapting core professional competencies to specific contexts, while drawing on global knowledge. To advance third-generation reforms, the Commission puts forward a vision: all health professionals in all countries should be educated to mobilise knowledge and to engage in critical reasoning and ethical conduct so that they are competent to participate in patient and population-centred health systems as members of locally responsive and globally connected teams. The ultimate purpose is to assure universal coverage of the highquality comprehensive services that are essential to advance opportunity for health equity within and between countries."

6 O AprenderSUS é destinado aos estudantes de graduação, conforme o Ministério da Saúde. Consultar o site oficial <http://portal.saude.gov.br/portal/arquivos/pdf/aprendersus.pdf>.

7 O VerSUS é destinado aos estudantes de graduação, conforme o Ministério da Saúde. Consultar o site oficial <http://portal.saude.gov.br/portal/sgtes/visualizar_texto.cfm?idtxt= 22371\#>.

$8 \mathrm{Na}$ aprendizagem ativa, conforme Bonnell e Eison (1991), os alunos ficam mais envolvidos quando existe algo mais do que só ouvir. Coloca-se menos ênfase na transmissão de informações e mais no desenvolvimento das habilidades dos alunos. Os estudantes se envolvem em pensamento de nível superior (análise, síntese, avaliação) e se engajam em atividades (por exemplo, leitura, discussão, escrita). Coloca-se maior ênfase na exploração que os estudantes fazem de suas próprias atitudes e de seus próprios valores.

9 Consultar grades curriculares dos cursos disponíveis nos sítios das universidades citadas: USP, grade curricular da Faculdade de Medicina: <https://uspdigital.usp.br/ jupiterweb/listarGradeCurricular? codcg $=5 \& \operatorname{cod} \operatorname{cur}=5042 \& \operatorname{codh} \mathrm{b}=0 \&$ tipo $=\mathrm{N}>$; UFMG, Projeto Pedagógico do curso de graduação em Enfermagem: <http://www.enf.ufmg.br/ site_novo/modules/mastop_publish/files/files_4c122f5142d80.pdf $>$. 


\section{Referências}

ALMEIDA, Márcio (Org.). Diretrizes curriculares nacionais para os cursos universitários da área da saúde. Londrina: Rede Unida, 2003.

BONNELL; Charles C.; EISON, James A. Active Learning: Creating Excitement in the Classroom. Higher Education Report, ASHE-ERIC, Washington DC, n. 1, 1991.

BRASIL. Presidência da República. Casa Civil. Subchefia para Assuntos Jurídicos. Constituição da República Federativa do Brasil de 1988. Art. 200, inciso III. Brasília, DF, 1988. Disponível em: <http://www. planalto.gov.br/ccivil_03/Constituicao/ Constituicao.htm>. Acesso em: 23 jan. 2013.

Ministério da Educação. Conselho Nacional de Educação. Câmara de Educação Superior. Resolução CNE/CES n. 4, de 7 de novembro de 2001. Disponível em: <http:// portal.mec.gov.br/cne/arquivos/pdf/ CES03.pdf>. Acesso em: 25 ago. 2011.

Ministério da Educação. Conselho Nacional de Educação. Câmara de Educação Superior. Resolução CNE/CES n. 4, de 7 de novembro de 2001. Institui Diretrizes Curriculares Nacionais do Curso de Graduação em Medicina. Brasília, DF, 2001. Disponível em: <http://portal.mec.gov.br/cne/arquivos/ pdf/CES04.pdf.>. Acesso em: 23 jan. 2013.

Ministério da Saúde. Politica de formação e desenvolvimento para o SUS: caminhos para a Educação Permanente em Saúde. Brasília: Ministério da Saúde, 2003.

Ministério da Saúde. Pró-saúde: programa nacional de reorientação da formação profissional em saúde. Brasília: Ministério da Saúde, 2005. 77 p. (Série C. Projetos, Programas e Relatórios).

CECCIM, Ricardo B.; FEUERWERKER, Laura C. M. O Quadrilátero da Formação para a Área da Saúde: Ensino, Gestão, Atenção e Controle Social. Physis: Revista de Saúde
Coletiva, Rio de Janeiro, v. 14, n. 1, p. 41$65,2004 a$.

Mudança na graduação das Profissões de saúde sob o eixo da integralidade. Cadernos de Saúde Pública, Rio de Janeiro, v. 20, n. 5, p. 1400-1410, set./out. 2004b.

CECCIM, Ricardo B.; BILIBIO, Luiz F. S. Articulação com o movimento estudantil da área da saúde: uma estratégia de inovação na formação de recursos humanos para o SUS. In: FERLA, Alcindo A.; FAGUNDES, Sandra M. S. (Org.). Tempo de inovações: a experiência da gestão da saúde do Rio Grande do Sul. Porto Alegre: Dacasa, 2002. p. 163-174.

CECCIM, Ricardo B.; CARVALHO, Yara M. de. Ensino da saúde como projeto da integralidade: a educação do profissionais do SUS. In: PINHEIRO, Roseni; CECCIM, Ricardo B.; MATTOS, Ruben A. de (Org.). Ensinar saúde: a integralidade e o SUS nos cursos de graduação na área da saúde. 2. ed. Rio de Janeiro: IMS/Uerj: Cepesc: Abrasco, 2006. p. 69-92.

CECCIM, Ricardo B.; CAPOZZOLO, Angela A. Educação dos profissionais de saúde e afirmação da vida: a prática clínica como resistência e criação. In: MARINS, João J. N.; REGO, Sergio; LAMPERT, Jadete B.; ARAÚJO, José G. C. de (Org.). Educação médica em transformação: instrumentos para a construção de novas realidades. São Paulo: Hucitec/Abem, 2004. p. 346-385.

FERREIRA, José R.; BUSS, Paulo M. O que o desenvolvimento local tem a ver com a promoção da saúde? In: ZANCAN, Lenira, et al. (Org.). Promoção da saúde como caminho para o desenvolvimento local: experiência em Manguinhos, RJ. Rio de Janeiro: Abrasco/Fiocruz, 2002.

FLEXNER, Abraham. Medical Education in the United States and Canada. New York: Carnegie Foundation for The Advancement of Teaching, 1910. (Bulletin, 4). 
FREIRE, Paulo. Pedagogia da autonomia: saberes necessários à prática educativa. 43. ed. São Paulo: Paz e Terra, 2011.

FRENK, Julio; CHEN, Lincoln. Health Professionals for a New Century: Transforming Education to Strengthen Health Systems in an Interdependent orld. The Lancet, London, v. 376, n. 9756, p. 1923-1958, dec. 2010.

HADDAD, Ana E. et al. Formação de profissionais de saúde no Brasil: uma análise no período de 1991 a 2008. Revista de Saúde Pública, São Paulo, v. 44, n. 3, p. 383-393, Jun. 2010.

MATTOS, Ruben de A. Integralidade, trabalho, saúde e formação profissional: algumas reflexões críticas feitas com base na defesa de alguns valores. In: MATTA, Gustavo C.; LIMA, Júlio C. F. (Org.). Estado, sociedade e formação profissional em saúde: contradições e desafios em 20 anos de SUS. Rio de Janeiro: Editora Fiocruz/EPSJV, 2008. p. 313-352.

PAGLIOSA, Fernando L.; DAROS, Marco A. O relatório Flexner: para o bem e para o mal. Revista Brasileira de Educação Médica, Rio de Janeiro, v. 32, n. 4, p. 492-499, 2008.
PEREIRA, Isabel B. A educação dos trabalhadores da saúde sob a égide da produtividade. In: MATTA, Gustavo C.; LIMA, Júlio C. F. (Org.). Estado, sociedade e formação profissional em saúde: contradições e desafios em 20 anos de SUS. Rio de Janeiro: Editora Fiocruz/EPSJV, 2008. p. 393-420.

PINHEIRO, Roseni; CECCIM, Ricardo B.; MATTOS, Ruben A. de (Org.). Ensinar saúde: a integralidade e o SUS nos cursos de graduação na área da saúde. Rio de Janeiro: IMS/ Uerj/Cepesc/Abrasco, 2005.

REDE INTERNACIONAL DE EDUCAÇÃO DE TÉCNICOS EM SAÚDE: RETS. Saúde no século XXI: novos desafios devem definir os rumos da formação. Rio de Janeiro, ano 3, n. 9, p. 2-11, jan./fev./mar. 2011.

ROSSONI, Eloá; LAMPERT, Jadete. Formação de profissionais para o Sistema Único de Saúde e as diretrizes curriculares. Boletim da Saúde, Porto Alegre, v. 18, n. 1, jan./ jun. 2004.

TOMEY, Agustín V. Abraham Flexner: pionero de la educación médica. Revista Cubana Educação Médica Superior, [s/l], v. 16, n. 2, p. 156-63, abr./jun. 2002.

Recebido em 26/04/2012

Aprovado em 01/10/2012 